The incidence of treatmentemergent adverse events (TEAE) was not significantly different between the four trial groups. Grade 1 and grade 2 TEAEs occurred in $20.5 \%$ and $5.0 \%$ of patients, respectively. Two grade 3 TEAEs and no grade 4 events were reported. None of the TEAEs was considered to be GV1001 related.

To date, the exact pathways through which GV1001 treatment results in beneficial effects in patients with BPH remain unclear and further laboratory and clinical studies are required to elucidate these mechanisms. In the future, if the agent becomes available on the market, the extended dosing regimen of GV1001 might be more convenient for patients than the daily administration required for most current drugs.

\section{Clemens Thoma}

ORIGINAL ARTICLE Moon, K. T. et al.

A randomised, placebo-controlled, multicentre, phase 2 clinical trial to evaluate the efficacy and safety of GV1001 in patients with benign prostatic hyperplasia. BJU Int. https://doi.org/10.1111/ bju.14233 (2018)

FURTHER READING Graham, M. K. et al. Telomeres and telomerase in prostate cancer development and therapy.Nat. Rev. Urol. 14, 607-619 (2017)

rats and, most importantly, reduced PVR after bPNI. As expected, no effect was observed in Trpv $4^{-/-}$rats, demonstrating the selectivity of the agonist and confirming that the effect was TRPV4-mediated. Bladder contractility, which was investigated by assessing the effect of carbachol on bladder strips, was significantly reduced in bPNI rats compared with controls. Contractions of bladder strips induced by GSK1016790A in vitro were small, suggesting a limited local role for TRPV4 in detrusor contractility.

Overall, the data suggest that increased afferent signalling via TRPV4 activation increases voiding frequency and reduces PVR after $\mathrm{PNI}$, but does not restore contractility of the detrusor muscle. "Whether these results hold true in other DUA models or in a patient population remains elusive," Everaerts concludes.

Annette Fenner

ORIGINAL ARTICLE Deruyver, Y. et al.

Intravesical activation of the cation channel TRPV4 improves bladder function in a rat model for detrusor underactivity. Eur. Urol. https://doi. org/10.1016/j.eururo.2018.05.020 (2018)

\title{
Diagnostic microfluidic immunoassays - go with the flow
}

Owing to the inadequate sensitivity and specificity of current bladder cancer detection methods such as urine cytology and cytoscopy, noninvasive and robust diagnostic techniques have been sought. Now, a new study published in Cancer Research reports the development of a novel microfluidic immunoassay for the detection of urinary-exfoliated tumour cells (UETCs) from voided urine of patients with bladder cancer that exhibits a higher sensitivity and specificity than other methods.

The authors developed a size-based microfluidic chip - containing 20,000-80,000 capture units - to efficiently capture UETCs of various sizes (individual cells or clusters) with intact cellular morphology. "Once the UETCs are captured, we used our specially concocted panel of biomarkers to correctly identify them," explains lead investigator Ray Han. Indeed, an on-chip, quantitative immunofluorescence assay based on combined expression of cytokeratin 20 (CK20) and CD44 variant 6 (CD44v6), which are overexpressed and associated with aggressive biology in urothelial tumours, was used to detect UETCs. Importantly, this size-based technique offers advantages over other immunoaffinitybased methods, which can only capture limited cell subsets that are not representative of the whole tumour owing to the use of a specific capture antibody.

The clinical validity of the microfluidic immunoassay was assessed in a cohort of 79 patients with bladder cancer and 43 agematched control individuals. Expectedly, $\mathrm{CK} 20^{+} \mathrm{CD} 44 \mathrm{v} 6{ }^{+}$UETC counts were significantly higher in the bladder cancer group than in the control group (53.3 versus $0.0, P<0.0001$ ).

Higher counts were also observed in patients with grade 2 or 3 bladder cancer than in those with grade 1 disease (485.7 versus $13.3, P<0.0001$ ), and in patients with tumours $>2 \mathrm{~cm}$ in size than in those with smaller tumours (133.3 versus 17.3, $P<0.0001)$. Notably, a 10 -fold cross validation of training data from a receiver operating characteristic (ROC) analysis revealed that the microfluidic immunoassay achieved an accurate bladder cancer diagnosis with an overall predictive accuracy of $0.85(95 \% \mathrm{Cl} 0.76-0.93)$, and with a sensitivity of $89.8 \%(95 \% \mathrm{Cl} 71.5-86.4)$ and a specificity of $71.5 \%(95 \% \mathrm{Cl} 59.7-83.3)$.

A paired comparison between the microfluidic immunoassay, traditional cytology, and two commercially-available proteomic tests (BTA stat and NMP22 BladderChek) was also performed in

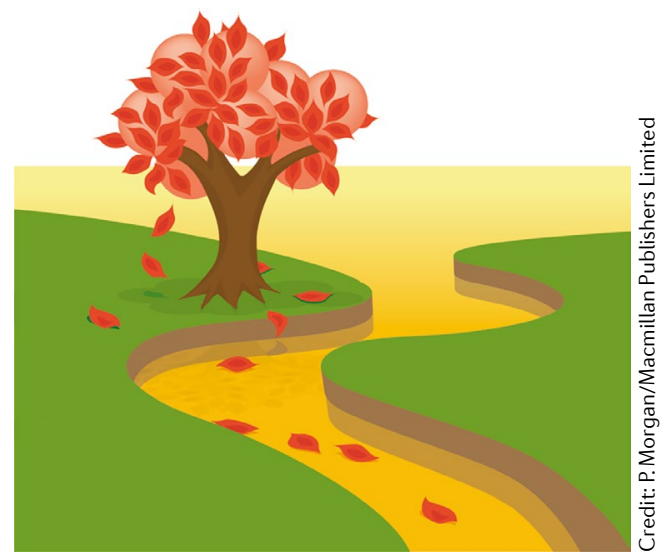

randomly-enrolled patients with bladder cancer and control individuals (both $n=20$ ). Strikingly, the microfluidic immunoassay had a higher overall sensitivity than other methods, particularly across tumour grades, and a sufficiently high level of specificity that was comparable to NMP22

BladderChek, but evidently lower than the perfect specificity of cytology.

"Tumour cells captured via size-based methods have a higher degree of cell viability, and this intact cellular morphology facilitates the pursuit of a downstream molecular analysis," explains Han. Indeed, off-chip, single-cell whole-genome sequencing and copy number alteration (CNA) analysis revealed that $91.7 \%$ of $C K 20^{+} \mathrm{CD} 44 \mathrm{v} 6^{+}$ UETCs possessed genomic instability and harboured CNAs, with a considerable degree of inter-UETC heterogeneity, highlighting the amenability of this technique for downstream profiling.

Overall, the findings demonstrate the promise of a novel microfluidic immunoassay for bladder cancer detection, and represent a framework upon which diagnostic performance could be further improved. "A better understanding of the single-cell sequencing of UETCs might produce a more robust form of 'liquid biopsy' in urine," says Han. "Going forward, we plan to seek further clinical validation involving larger trials, automate the whole procedure using an integrated device, and interrogate more genomic information from both single UETCs and paired primary tumour tissues."

Conor A. Bradley

ORIGINAL ARTICLE Chen, A. et al. Detection of bladder cancer via microfluidic immunoassay and single-cell DNA copy number alteration analysis of captured urinary exfoliated tumor cells. Cancer Res. https://doi.org/10.1158/0008-5472.CAN-17-2615 (2018) 